\title{
OCORRÊNCIA DE RHOPALURUS DEBILIS (C.L. KOCH, 1840) (SCORPIONES, BUTHIDAE) NO ESTADO DA BAHIA, BRASIL
}

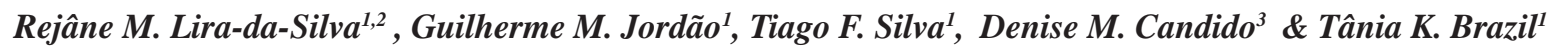

Biota Neotropica v5 (n1a) http://www.biotaneotropica.org.br/v5n1a/pt/abstract?short-communication+bn014051a2005

Recebido em 07/12/2003

Publicado em 01/02/2005

${ }^{1}$ Núcleo Regional de Ofiologia e Animais Peçonhentos da Bahia (NOAP). Campus Universitário de Ondina, Universidade Federal da Bahia, Instituto de Biologia, Salvador, Brasil, 40.170-210.

E-mails: gmjordao@ufba.br; tiagofs@ufba.br; rejane@ufba.br; taniabn@ufba.br. ${ }^{2}$ Faculdade de Tecnologia e Ciências (FTC). Av. Luís Viana Filho, 8812, Salvador, Brasil, 41.820-785.

E-mail: rejane.ssa@ftc.br

${ }^{3}$ Instituto Butantan. Av. Vital Brazil, nº 1500, São Paulo, SP, Brasil, 05503-900.E-mail: denisecandido@butantan.gov.br.

\begin{abstract}
This work reports the occurrence of Rhopalurus debilis (C.L. Koch, 1840), in the state of Bahia, Brazil, adding a species to its escorpiofauna and highlighting the importance of carrying out regional studies on these animals. Seven scorpions ( 2 males, 4 females and 1 young) were collected in the city of Curaçá $\left(08^{\circ} 59^{\circ} 31^{\circ} \mathrm{S} / 39^{\circ} 54^{\circ} 29^{\circ} \mathrm{W}\right)$, from March $14^{\text {th }}$ to $16^{\text {th }}, 2003$. They were recorded in the Núcleo Regional de Ofiologia e Animais Peçonhentos da Bahia (NOAP) and included in the Scientific Collection of Scorpions of the Federal University of Bahia. These specimens represent a new record of this species in the right riverbank of the São Francisco river.
\end{abstract}

Key words: Distribution, Scorpion, Rhopalurus debilis, Bahia, Brazil.

\section{Resumo}

Este trabalho assinala a ocorrência de Rhopalurus debilis (C.L. Koch, 1841), no estado da Bahia, Brasil, acrescentando mais uma espécie à sua escorpiofauna e reforçando a importância do estudo regionalizado desses animais. Sete escorpiões (2 machos, 4 fêmeas e 1 jovem) foram coletados no município de Curaçá (08 59’31"S/ 39 54’ 29"W), entre os dias 14 e 16 de março de 2003, registrados no Núcleo Regional de Ofiologia e Animais Peçonhentos da Bahia (NOAP) e tombados no Museu de Zoologia da Universidade Federal da Bahia. Este registro revela uma nova ocorrência desta espécie, na margem direita do rio São Francisco.

Palavras-chave: Distribuição, Escorpião, Rhopalurus debilis, Bahia, Brasil.

http://www.biotaneotropica.org.br 


\section{INTRODUÇÃO}

Apesar dos escorpiões serem considerados como um grupo numericamente pouco expressivo, abrangem cerca de 1500 espécies, distribuídas globalmente em 14 famílias e 163 gêneros (Soleglad \& Fet 2003). A ordem Scorpiones apresenta ampla distribuição geográfica, estando representada em todos os continentes, com exceção da Antártida. No Brasil, a escorpiofauna é representada por cinco famílias, Liochelidae, Euscorpiidae, Chactidae, Bothriuridae e Buthidae, abrangendo 17 gêneros (Soleglad \& Fet 2003) e 86 espécies consideradas atualmente válidas (Lourenço \& Eickstedt 2003).

A Bahia apresenta escorpiofauna diversificada com o registro de 18 espécies nesta região, cerca de 25\% ou um quarto da diversidade de escorpiões brasileiros, agrupadas nas famílias Bothriuridae e Buthidae. A primeira com pelo menos 2 espécies (Bothriurus asper Pocock, 1893 e B. rochai Mello-Leitao, 1932) e a segunda com 16 espécies (Ananteris balzanii Thorell, 1891; Isometrus maculatus (De Geer, 1778); T. bahiensis (Perty, 1833); T. brazilae Lourenço \& Eickstedt, 1984; T. lamottei Lourenço, 1997; T. mattogrossensis Borelli, 1901; T. melici Lourenço, 2003; T. neglectus Mello-Leitão, 1932; T. pusillus Pocock, 1893; T. stigmurus (Thorell, 1876); T. serrulatus Lutz \& Mello, 1922; Rhopalurus agamenon (C. L. Koch, 1840); R. acromelas Lutz \& Mello, 1922; R. rochai Borelli, 1910; $R$. debilis (C. L. Koch, 1840) e $R$. lacrau Lourenço \& Pinto-da-Rocha, 1997). Entre os botriurideos, provavelmente, serão acrescidas mais 5 espécies que estão sendo descritas como trabalho de tese de doutorado por Camilo Mattoni, da Universidad Nacional de Córdoba, Argentina (comunicação pessoal, 2003).

A observação de escorpiões no município de Curaçá no Estado da Bahia, foi notificada pelos pesquisadores do Centro de Pesquisas Gonçalo Muniz (Fundação Osvaldo Cruz - FIOCRUZ/Bahia), Dr. François Noireau e MsC. Artur Dias Lima ao NOAP, durante expedição científica relativa ao estudo dos barbeiros (Insecta; Triatominae) na região, com o objetivo de identificar os possíveis fatores que favorecem a interação desses aracnídeos com os insetos pesquisados pela equipe da FIOCRUZ. A análise do material coletado permitiu reconhecer e relatar a ocorrência do $R$. debilis no território do Estado da Bahia, em Curaçá (08 59’ 31"S/ 39º 54’ 29"W) e acrescentando, portanto, mais uma espécie à escorpiofauna baiana.

\section{MATERIAL E MÉTODOS}

Sete exemplares foram coletados entre os dias 14 e 16 de março de 2003, no município de Curaçá, localizado no Estado da Bahia, na região do baixo-médio São Francisco, fazendo divisa com o Estado de Pernambuco (Fig. 01). Este município é caracterizado por apresentar uma vegetação de Savana Estépica (caatinga do sertão-árido), solo predominantemente com horizonte B textural, apresentando relevo com depressões periféricas e interplanálticas e um clima árido e semi-árido (Secretaria do Planejamento do Estado da Bahia 2003).

Utilizou-se um esforço de coleta de quatro dias (8 horas/dia), com uma equipe de cinco pessoas, investigandose os microhabitats de peridomicílio, ocos de árvores, ninhos de aves e fendas de pedras.

Para coletar os animais em ocos de árvores, utilizou-se um machado para abrir os ocos e com auxílio de uma pinça os animais foram acondicionados em tubos de vacutainer. Para os ninhos de aves abandonados, foi utilizado um gancho para derrubá-los sobre um guarda-chuva entomológico (1,20m X $1,50 \mathrm{~m})$, e, com auxílio de uma pinça os animais foram acondicionados em tubos de vacutainer.

Os animais foram conservados em álcool glicerinado a 70\%, medidos com um paquímetro digital e observados em lupa, para a análise taxonômica. Todos os exemplares foram tombados na coleção científica de escorpiões do Museu de Zoologia da Universidade Federal da Bahia.

\section{RESULTADOS E DISCUSSÃO}

Os escorpiões foram encontrados em ocos de árvores $(n=4)$ e ninhos de aves como o carcará (Caracara plancus (Miller)), o casaca-de-couro (Pseudoseisura cristata (Spix)) e a alma-de-gato (Piaya cayana (Linnaeus)) ( $n=3$, respectivamente), locais que lhes servem como abrigo, já que preservam a umidade e são também habitat de outros artrópodes, base alimentar dos escorpiões (Brownel \& Polis 2001). Os sete exemplares (2 machos, 4 fêmeas e 1 jovem) foram registrados no Núcleo Regional de Ofiologia e Animais Peçonhentos da Bahia, Instituto de Biologia, UFBA, e tombados na coleção científica de escorpiões da Universidade Federal da Bahia (UFBA-esc 1582 a 1588).

R. debilis (Fig. 2) foi redescrita por Borelli (1910) a partir de um exemplar fêmea coletado no Ceará. Sua distribuição abrange ainda os Estados de Pernambuco e Paraíba (Fet et al. 2000). É caracterizada por apresentar cefalotórax amarelo, com um grande triângulo anterior denegrido e com bordas laterais esmaecidas, metassoma dilatando-se regularmente para trás, segmentos I ao III amarelados e V marrom avermelhado, com cristas medianas dorsais completas e presentes nos segmentos I a IV, levemente denticuladas, com o último dente um pouco maior (Mello-Leitão 1945) sendo que o tamanho dos exemplares adultos coletados em Curaçá variou de 16,00-28,26mm, o que diverge dos 23-30mm propostos por Lourenço (1982). Quatro dos cinco espécimes adultos mediram abaixo de 23mm. O número de dentes nos pentes variou de 15-17, diferindo também dos dados propostos por Lourenço (1982) que aponta 12-16.

A ocorrência desta espécie no ambiente morfoclimático da caatinga, característico do município de Curaçá, corrobora os dados de Lourenço (1982), que descreve o $R$. debilis como sendo característico deste ambiente, porém, revela um dado novo que é a ocorrência deste animal na 
margem direita do rio São Francisco. Este rio banha as terras do Estado de Minas Gerais e divide os Estados da Bahia, Pernambuco, Sergipe e Alagoas. O São Francisco é o mais extenso rio brasileiro e liga as regiões Sudeste e Nordeste, com seus 2.700 quilômetros e largura de $2 \mathrm{~m}$ a $25 \mathrm{~km}$, características estas que o faz importante barreira geográfica natural, o que pode levantar importantes hipóteses futuras sobre a biogeografia da espécie.

\section{AGRADECIMENTOS}

Os autores agradecem à equipe do Dr. François Noireau, que, juntamente com Artur Dias Lima permitiram ao segundo autor acompanhar as atividades do Projeto, e, à pesquisadora do Instituto Butantan, Dra. Sylvia Lucas, pela orientação e cuidados na discussão do trabalho.

\section{REFERÊNCIAS BIBLIOGRÁFICAS}

BROWNELL, P. \& POLIS, G.A. . 2001. Scorpion Biology and Research, Oxford University.

FET, V., SISSOM, W. D., LOWE, G. \& BRAUNWALDER, M. E.. 2000. Catalog of the Scorpions of the World (17581998). New York: New York Entomological Society, 690.

LOURENÇO, W.R. 1982. Révision du genre Rhopalurus Thorell, 1876 (Scorpiones, Buthidae). Rev. Arachnol. 4: $107-141$.

LOURENÇO, W.R. \& EICKSTEDT, V.R.D. VON 2003. Escorpiões de Importância Médica. In Cardoso, J.L.C. et al. Animais Peçonhentos no Brasil. Biologia, Clínica e Terapêutica dos Acidentes. SARVIER, São Paulo. 468 p.

MELLO-LEITÃO, C.F. DE. 1945. Escorpiões Sul Americanos. Arquivos do Museu Nacional, 40: 1-468.

SECRETARIA DO PLANEJAMENTO, CIÊNCIA E TECNOLOGIA. 2003. Anuário Estatístico da Bahia. 16: 63-93.

SOLEGLAD, M.E. \& FET, V. 2003. High level systematics and phylogeny of the extant scorpions (Scorpiones: Orthosterni). Euscorpius, 11: 1-175.

Título: Ocorrência de Rhopalurus debilis (C.L. Koch, 1840) (Scorpiones, Buthidae), no estado da Bahia, Brasil.

Autores: Rejâne M. Lira-da-Silva, Guilherme M. Jordão, Tiago F. Silva, Denise M. Candido \& Tânia K. Brazil

Biota Neotropica, Vol. 5 ( número1a): 2005

http://www.biotaneotropica.org.br/v5n1a/pt/ abstract?short-communication+bn014051a2005

Recebido em 07/12/2003 - Publicado em 01/02/2005

ISSN 1676-0603

http://www.biotaneotropica.org.br 